\title{
GLASS: The Grism Lens-Amplified Survey From Space. HST Grism Spectroscopy of the Frontier Fields.
}

\author{
Kasper B. Schmidt and The GLASS Team \\ Department of Physics, University of California, Santa Barbara, CA, 93106-9530, USA
}

\begin{abstract}
The Grism Lens-Amplified Survey From Space (GLASS) is a 140 orbit spectroscopic survey of 10 massive galaxy clusters, including the six Hubble Frontier Fields. GLASS has observed the cluster cores with the HST-WFC3 G102 and G141 grisms providing a wide wavelength coverage in the near-infrared from roughly $0.8-1.7 \mu \mathrm{m}$. The parallel fields were observed through the optical ACS G800L grism. Taking advantage of the lensing magnification of the clusters, GLASS reaches intrinsic spectroscopic $1 \sigma$ flux limits of roughly $10^{-18} \mathrm{erg} / \mathrm{s} / \mathrm{cm}^{2}$ and improved spatial resolution for lensed sources behind the clusters. These features are particularly useful for the three main science drivers of GLASS which are, I) exploring the universe at the epoch of reionization, II) describe how metals cycle in and out of galaxies, and III) asses the environmental dependence of galaxy evolution. The former two benefit highly from the improved depth and increased resolution provided by the cluster lensing. Apart from the main science drivers, a slew of ancillary science has been enabled by the survey, including improving cluster lens modeling and searches for supernovae. Here we present the survey and the GLASS data releases, which are continuously being made available to the community through https://archive.stsci.edu/prepds/glass/. For further information we refer to Schmidt et al. (2014), Treu et al. (2015), and http://glass.physics.ucsb.edu.
\end{abstract}

Keywords. surveys, infrared: galaxies, techniques: spectroscopic, galaxies: clusters: general, galaxies: evolution, galaxies: high-redshift, galaxies: structure, line: identification

Survey Overview: The data acquisition of the Grism Lens-Amplified survey from Space (GLASS) presented by Schmidt et al. (2014) and Treu et al. (2015) was completed in January 2015. The data include 140 orbits of HST grim spectroscopy of the 10 massive galaxy clusters A370 ${ }^{\dagger}, \mathrm{A} 2744^{\dagger}, \mathrm{MACS}^{\circ} 416^{\star \dagger}, \mathrm{MACS}^{\circ} 717^{\star \dagger}, \mathrm{MACS}^{\star} 0744^{\star}, \mathrm{MACS}^{\star} 149^{\star \dagger}$, MACS1423^, MACS2129^, RXJ1347 ${ }^{\star}$, and RXJ2248 ${ }^{\star} \dagger$. Here ${ }^{\star}$ designates a CLASH cluster (Postman et al., (2012)) and ${ }^{\dagger}$ designates Hubble Frontier Fields clusters (P.I. J. Lotz). The cluster cores were observed through the two near-infrared grisms G102 and G141 on HST's WFC3, whereas the parallel fields were observed through the optical ACS grism G800L. The slitless HST grisms provide three-dimensional spectroscopy with a spectral resolution of $\sim 100-200$ and spatial information at the native $H S T$ revolution of each object and its potential emission lines. Each of the GLASS clusters was observed at two different position angles roughly 90 degrees apart. The GLASS observing strategy is exemplified by the layout of the MACS0717 data shown in Figure 1. This was done to optimize disentangling and accounting for contaminating flux from neighboring objects in the slitless grism spectroscopy of the crowded clusters, as well as to improve emission line detection and identification as illustrated in Figure 2. At each position angle the GLASS spectroscopy reaches observed $1 \sigma$ flux limits of roughly $5 \times 10^{-18} \mathrm{erg} / \mathrm{s} / \mathrm{cm}^{2}$. Combining the two individual spectra at the two orientations improves this limit by a factor $\sqrt{2}$ for all objects in the GLASS field-of-view. Furthermore, these flux limits have not been corrected for the lensing magnification from the foreground cluster, $\mu$. Hence, background 

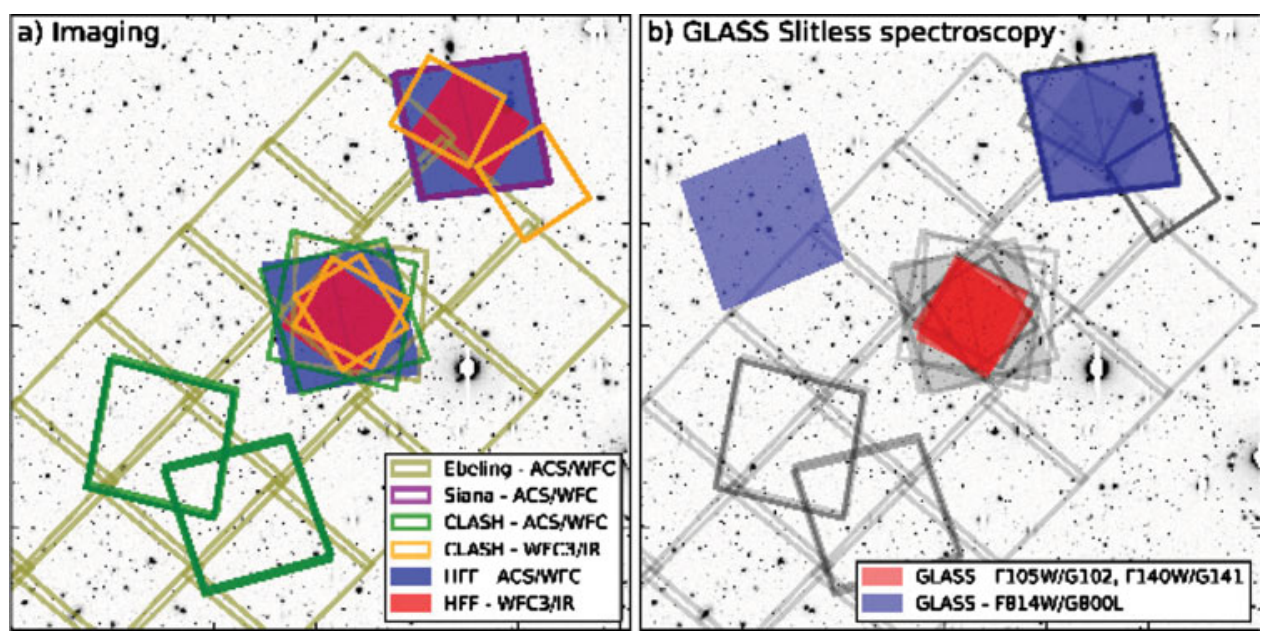

Figure 1. The layout of the available ancillary data of MACS0717 (left panel). The right panel illustrates the observing strategy of GLASS: Each of the 10 clusters are observed at two distinct position angles roughly 90 degrees apart. The near-infrared WFC3 grisms G102 and G141 are put on the cluster cores, whereas the two parallel fields are observed with the ACS G800L grism. One of the ACS parallel is aligned with the CLASH and/or HFF parallel fields (left panel). Having two distinct position angles of data on the cluster core optimizes the disentailing and accounting for contaminating flux from neighboring sources, and improves emission line detection and identification as illustrated in Figure 2. Figure taken from Treu et al. (2015).

sources will have intrisic flux limits a factor $\mu$ deeper, enabling spectroscopy of otherwise un-observable targets. The cluster lensing also improves the spatial resolution of background source, enabling spatially resolved studies of samples of galaxies at $z>1$. These three key aspects of the GLASS data, i.e. the three-dimensional spectral information, the lens-amplified spatial resolution of background sources, and the lens-improved spectroscopic depths, are crucial for the GLASS science drivers.

GLASS Science Drivers: The first science driver of GLASS is to study and explore the Universe at the epoch of reionization. We will use the hundreds of spectra of galaxies at $z>6$ to shed light on the epoch of reionization, the role these galaxies play in reionizing the universe, and the Ly $\alpha$ escape fraction at the cosmic dawn. The first study addressing these topics was presented by Schmidt et al. (2015). Here we search for Ly $\alpha$ emission in more than 150 galaxies with photometric selection redshifts of $z \gtrsim 7$. These were assembled from 6 of the 10 GLASS clusters including the three Frontier Fields clusters A2744, MACS0717, and RXJ2248, using both Lyman break selection techniques and photometric redshifts. Visually inspecting the GLASS spectra of all objects we found more than 20 promising candidate emission line sources, with lines consistent with being Ly $\alpha$ at $z \gtrsim 7$. As the modest resolution of the HST grisms cannot resolve the asymmetry of the Ly $\alpha$ line, we are leading several high-resolution ground-based spectroscopic followup programs with both HST and VLT to confirm the high redshift nature of the sources. Assuming that the emission lines are $\operatorname{Ly} \alpha$, we stacked the sources in an attempt to recover emission from the rest-frame UV emission lines CIV and CIII]. In Figure 3 we show the stack of the most promising line emitters with $\langle z\rangle=7.2$ at rest-frame wavelengths around Ly $\alpha$, CIV, and CIII]. We do not detect any emission from CIV, and CIII], with estimated flux ratio limits of $f_{\mathrm{CIV}} /\left[f_{\mathrm{Ly} \alpha}\right] \lesssim 0.32$ and $\left.f_{\mathrm{CIII}}\right] /\left[f_{\mathrm{Ly} \alpha}\right] \lesssim 0.23$, respectively. These limits are in good agreement with the ones presented by, e.g., Shapley et al. (2003), Erb 


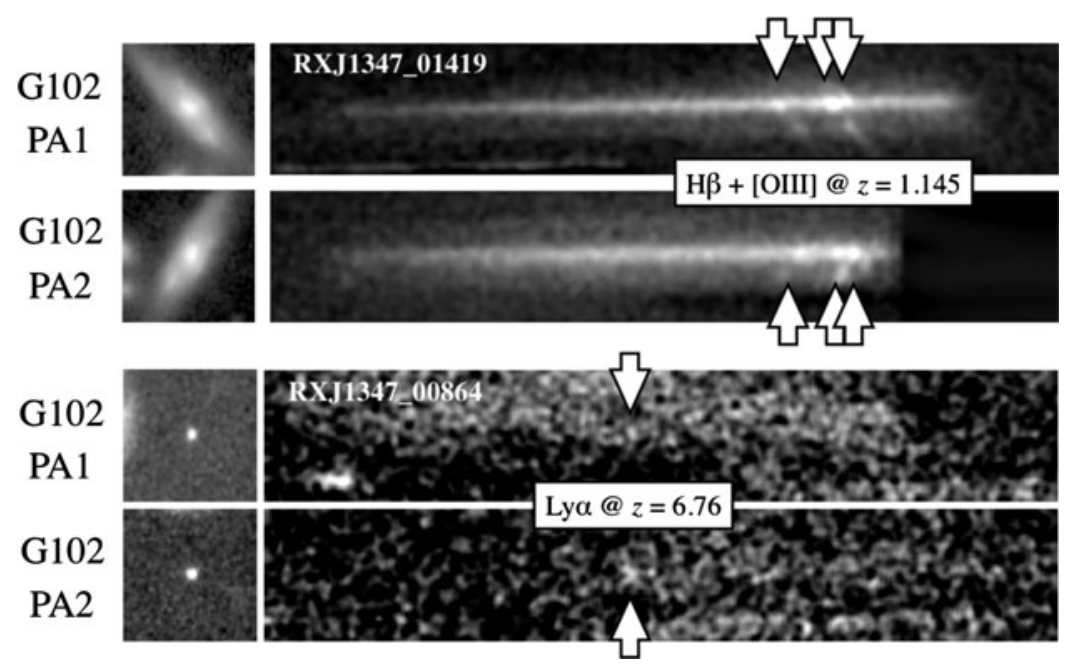

Figure 2. The GLASS G102 spectra of two emission lines sources from the GLASS RXJ1347 data release illustrating the power of the GLASS observing strategy (Figure 1). For the first source, RXJ1347_01419, it is trivial to determine the redshift as both [OIII] $\lambda \lambda 4959,5007 \AA$ and $\mathrm{H} \beta$ are cleanly detected and match the spatial near-infrared morphology of the direct images shown in the left. This source is included in the v001 GLASS data release available at https://archive.stsci.edu/prepds/glass/. The emission line in the spectra for the second source, RXJ1347_00864, on the other hand, is only marginal. In this case the second PA of the GLASS data is crucial in confirming this emission line to be real. This source is a confirmed Ly $\alpha$ emitter at $z=6.76$ as presented by Huang et al. (2015) and Schmidt et al. (2015). In the spectra of RXJ1347_00864 the levels of contamination (flux from neighboring objects) are distinctly different between the two GLASS spectra, optimizing the ability to account for this contamination.

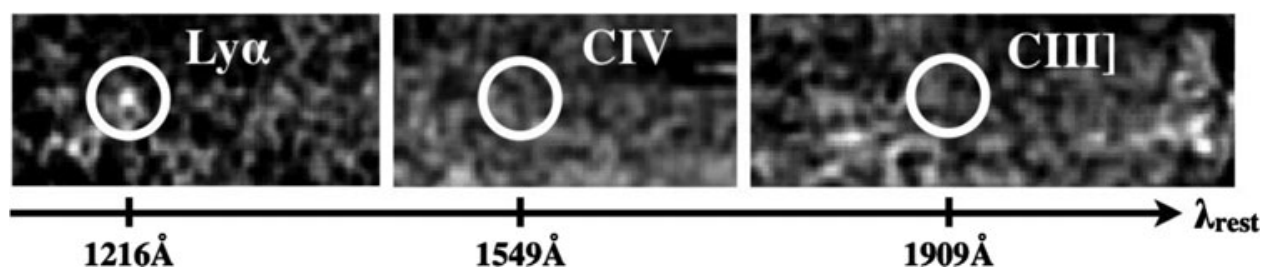

Figure 3. The rest-frame stack of promising Ly $\alpha$ emitters at $\langle z\rangle=7.2$. No CIV and CIII] emission is detected. The spatial profile (vertical direction) of the Ly $\alpha$ emission is comparable to that of the continuum UV emission. Any extended Ly $\alpha$ emission is below the surface brightness detection threshold in the stack. Figure taken from Schmidt et al. (2015).

et al. (2010) and Stark et al. (2014), Stark et al. (2015a), Stark et al. (2015b). The spatial information in the GLASS spectra allows an estimate of the spatial profile of the Ly $\alpha$ emission from the stack shown in Figure 3. We found that the extent of the emission is comparable in size to that of the continuum UV emission. Hence, any diffuse Ly $\alpha$ emission is below the surface brightness detection limit in the stack. We confirm this by comparing the GLASS detection threshold to stacks of galaxies simulated to be at $z=7.2$ from the Ly $\alpha$ Reference Sampled (LARS) presented by Hayes et al. (2013), Hayes et al. (2014), Östllin et al. (2014). For details on this study see Schmidt et al. (2015) and the proceeding for XXIXth IAU General Assembly Symposion 319.1.06.

The second science driver of GLASS is to study how metals cycle in and out of galaxies. From emission lines detected in the slitless GLASS spectra, we are mapping the spatially resolved star formation. This allows a study of the gas accretion, star formation, outflows, 
and a determination of the metallicity gradients in galaxies at $z \sim 2$, as shown by Jones et al. (2015) (see also proceeding for FM22.2.03).

The final main science goal of GLASS is to study how the environment affects galaxy evolution. We explore the environmental dependence of galaxy evolution using the first comprehensive census of spatially resolved star formation in dense environments, i.e., the cluster cores as well as the cluster infall regions. A first study using only the cluster core data has been presented by Vulcani et al. (2015) (see also proceeding for FM22.4.03).

Apart from the three main science drivers, a wide range of ancillary science has been enabled by the GLASS data. Two particularly interesting examples are the improvement of cluster lens models and the search for supernovae in the direct imaging taking immediately prior to each grism exposure. For the former, we published an updated lens model of A2744 in Wang et al. (2015) (see also proceeding for FM22.2.04), providing new galaxy redshifts to the community. For the latter example, we have been searching for supernova in the more than 40 individual GLASS visits, which resulted in the discovery of the first-ever multiple imaged supernova, SN Refsdal, as presented by Kelly et al. (2015) (see also proceeding for FM22.7.05).

Data Releases: The broad range of topics listed above illustrates the scientific potential of GLASS. To accommodate future studies, we are releasing the reduced data products from GLASS to the public via https://archive.stsci.edu/prepds/glass/ . To accompany these data releases and to provide a convenient and efficient way of inspecting and browse the GLASS data products, we have also released the Python-based GLASS inspection GUI (GiG) and the GLASS inspection GUI for redshifts (GiGz), which are available at https://github.com/kasperschmidt/GLASSinspectionGUIs and described in Treu et al. (2015).

In summary GLASS has already proven to be a fruitful and multi-facetted survey, with a wide range of scientific applications. With the ongoing release of the GLASS data products at https://archive.stsci.edu/prepds/glass/ we expect this to continue

\section{References}

Erb et al., 2010, ApJ, 719, 1168

Hayes et al., 2013, ApJ, 765, L27

Hayes et al., 2014, ApJ, 782, 6

Huang et al., 2015, ApJ submitted, arXiv:astro-ph:1504:02099

Jones et al., 2015, AJ, 149, 107

Kelly et al., 2015, Science, 347, 6226

Östllin et al., 2014, ApJ, 797, 11

Postman et al., 2012, ApJS, 199, 25

Schmidt et al., 2014, ApJ, 782, L36

Schmidt et al., 2015, ApJ submitted

Shapley et al., 2003, ApJ, 588, 65

Stark et al., 2014, MNRAS, 445, 3200

Stark et al., 2015a, MNRAS, 450, 1846

Stark et al., 2015b, MNRAS, submitted, arXiv:astro-ph:1504:06881

Treu et al., 2015, ApJ in press, arXiv:astro-ph:1509.00475

Vulcani et al., 2015, ApJ submitted

Wang et al., 2015, ApJ, 811, 29 